\title{
Enhanced Electro-Optic Modulation Performance in Optical Buffers by Slowing Light in Optimized Photonic Crystal Slab Structures
}

\author{
F. BAGCI AND B. AKAOGLU \\ Department of Engineering Physics, Faculty of Engineering, Ankara University, 06100 Besevler, Ankara, Turkey
}

\begin{abstract}
Slow light holds the key to advanced optical buffering and time-domain optical signal processing technologies. Photonic crystal based optical buffers are particularly attractive due to their nanoscale size, room temperature operation, and enhanced field dependent nonlinear response associated with the presence of slow light. In this study, the slow light and electro-optic modulation characteristics of a line-defect Si photonic crystal slab with triangular arrangement of holes filled with an electro-optic polymer $(n=1.6)$ are investigated by three-dimensional plane-wave expansion and finite-difference time-domain methods. The first rows adjacent to the line-defect are shifted gradually in the direction of light propagation and a slow light region with a high group index below the light-line is obtained for a shifting amount between $0.22 a$ and $0.27 a$. For the photonic crystal configuration with $0.22 a$ shifted rows, under modulated voltage change, the average group index is found to be decreasing with an increase in the bandwidth. The results show that the low group velocity supports a large delay time in a small modulated voltage variation. A linear change of group index with modulated voltage is obtained and the modulation sensitivity of central wavelength is obtained as $9.45 \mathrm{~nm} / \mathrm{V}$ for a delay line length of $0.5 \mathrm{~mm}$. Almost the same buffer capacity and bit length are found which provides the control of delay time flexibly while keeping the buffer capacity and the bit length almost unchanged.
\end{abstract}

DOI: $10.12693 /$ APhysPolA.125.611

PACS: 42.70.Qs, 42.79.Hp, 42.65.Pc

\section{Introduction}

An optical buffer is a device that temporarily stores and tunes the timing of optical signals. Optical delay lines and buffers constitute crucial components for future optical communication networks and information processing systems [1]. The most practical buffer systems consist of fiber delay lines with optical switches. However, these systems are very bulky to be handy in full size optical networks. On the other hand, compared with delay lines and buffers employing electromagnetically induced transparency and optical fibers, photonic crystal (PC) based systems are very compact for on-chip integration, operate at room temperature, show ultrafast response and provide tailorable bandwidth with low group velocity and dispersion-free propagation [2].

It is well known that slow light enhances nonlinear effects as a consequence of compression of local density of states [3]. The natural slow light occurring at the band edges of photonic crystals suffer large group velocity dispersion [4]. To overcome the large higher-order dispersion terms, a variety of approaches have been proposed [5-8].

In this study, a line-defect Si photonic crystal slab with nonlinear polymer $(n=1.6)$ [9] filled holes is proposed and slow light generation is employed by shifting the first rows adjacent to the line-defect in the direction of light propagation. The structure is optimized by scanning a wide range of shifting amounts and slow light region is obtained for average group indices between 69 and 339 for the shifts in the interval of $\delta x=0.22 a$ and $0.27 a$. First, slow light properties in $3 \mathrm{D}$ air-bridge photonic crystal slab with polymer infiltrated holes have been discussed. Second, local field effect enhancement induced by slow light transmission is analyzed and the amount of refractive index change in the structure under voltage application is calculated. Finally, slow light modulation by voltage application is analyzed in terms of frequency shifting and transmission control. Buffer performance under modulated voltage change is determined by a number of figure of merits, such as delay time, maximum storage capacity and physical size of the stored bit.

\section{Waveguide structure and modeling}

The investigated structure is a W1 line-defect PC slab structure consisting of triangular symmetry lattice holes filled with an electro-optic polymer (Fig. 1). The largest electro-optic coefficient of the polymer $\left(\gamma_{33}=150 \mathrm{pm} / \mathrm{V}\right)$ is used by placing the electrodes on each side of the waveguide [9]. The radius of holes is arranged as $0.30 a$ and the height of slab is chosen as $0.53 a$, where $a$ is lattice constant with $415 \mathrm{~nm}$. The rows adjacent to the line-defect are shifted in the direction shown by blue arrows in Fig. 1 to generate slow light. Band diagrams are obtained by using 3D plane-wave expansion method [10].

One of the important parameters in slow light research is group index $\left(n_{\mathrm{G}}\right)$, which is a measure of the slowness of light. $n_{\mathrm{G}}$ is defined as:

$$
n_{\mathrm{G}}=c \mathrm{~d} k / \mathrm{d} \omega .
$$

The average group indices and bandwidths are determined within the $10 \%$ range of the minimum group index in the constant $n_{\mathrm{G}}$ region. Since group index and bandwidth are inversely proportional, normalized delay-bandwidth products (NDBP), which are products of 


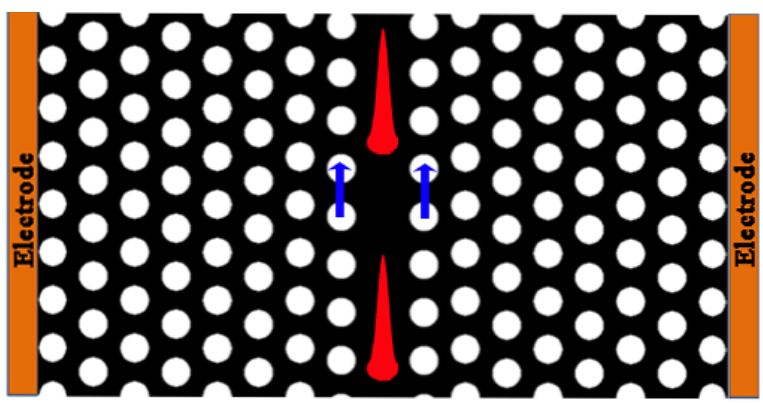

Fig. 1. The schematic structure of the line-defect photonic crystal slab with shifted first rows of holes. Shifted rows are remarked with blue arrows and the propagating pulse is shown in red.

group index and normalized bandwidth, are also calculated.

The nonlinear effects enhance with the decrease of group velocity, which induces an effective second-order susceptibility much greater than that for the bulk polymer. The relation is given as [11]:

$$
\chi_{\mathrm{PC}}^{\langle 2\rangle}=f^{3} \chi_{\mathrm{BULK}}^{\langle 2\rangle},
$$

where $\chi_{\mathrm{BULK}}^{\langle 2\rangle}$ is second order susceptibility in the bulk polymer, $f$ is the local-field factor. $f$ is given by [12]:

$$
f=n_{\mathrm{G}}^{\mathrm{PC}} / n_{\mathrm{G}}^{\mathrm{bulk}} \text {. }
$$

Due to the Pockels effect, the refractive index of electro-optic polymer shows a refractive index variation in the presence of external voltage. This variation is given as [11]:

$$
\Delta n=-\frac{1}{2} n_{\text {poly }}^{3} \gamma_{33} f^{3} \frac{U}{d} .
$$

Here $U$ represents the modulated voltage and $d$ is the distance between the electrodes $(d=6.42 \mu \mathrm{m})$.

There are important figure of merits pointing the performance of buffers, such as delay time, buffer capacity, and bit length. The delay time (storage time) is equal to $T_{\mathrm{s}}=L n_{\mathrm{G}} / c$, where $L$ is length of the photonic crystal based buffer, $c$ is light velocity in vacuum and $n_{\mathrm{G}}$ is average group index. The buffer capacity is given by the product of delay time and bandwidth. The capacity of the buffer is given as [13]:

$$
C=T_{\mathrm{s}} B=\frac{L}{2 a} n_{\mathrm{G}} \Delta \omega .
$$

Another important parameter is the physical size of the stored bit and it is calculated by $L_{\text {bit }}=L / C$.

\section{Results and discussions}

\subsection{Slow light performance of the photonic crystal slab}

The proposed line-defect PC slab supports one even and one odd mode in the TE-like photonic band gap (PBG) of $415 \mathrm{~nm}$ range. TE-even mode, which exists between $0.282(a / \lambda)$ and $0.263(a / \lambda)$ below the light line, is considered throughout the study to efficiently couple light into the structure.

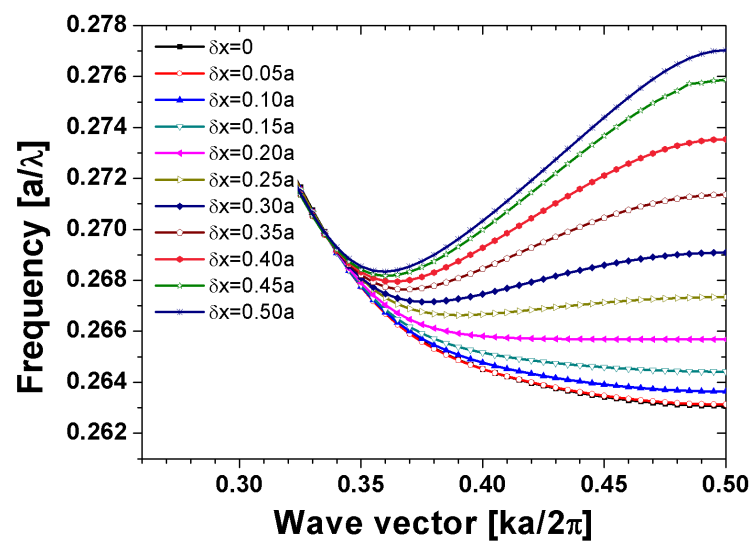

Fig. 2. Dispersion characteristics of the $\mathrm{PC}$ for $\delta x$ shifts from 0 to $0.5 a$.

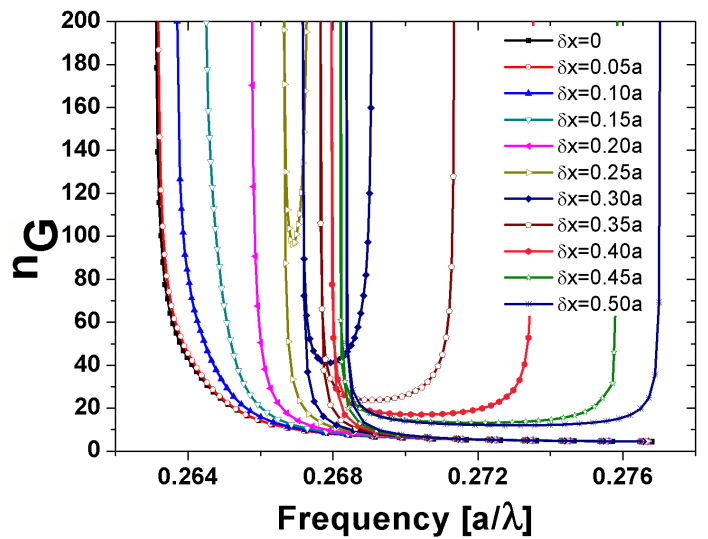

Fig. 3. Group index characteristics of the PC for $\delta x$ shifts from 0 to $0.5 a$.

Shifting of innermost rows is a very convenient method to generate slow light in terms of fabrication control $[7,8]$. We analyze the effects of shifting the rows of holes adjacent to the line-defect in the direction of light propagation, without changing the width of line-defect. The shifts are denoted with $\delta x$ letter. Since there is symmetry along the waveguide axis, $\delta x$ is only investigated from 0 to $0.5 a$. When there is no shift, guided mode consists of index-guided and gap-guided mode regions with a turning-point between them [5]. As the first rows shift, the frequencies of gap-guided mode increase, the mode becomes very flat for $\delta x=0.20 a$ and faces upwards for larger shifts (Fig. 2). The average $n_{\mathrm{G}}$ values decrease and bandwidths increase as $\delta x$ increases (Fig. 3).

The most promising region for slow light is obtained for the shifts in the interval of $\delta x=0.22 a$ and $\delta x=0.27 a$ (Fig. 4). The average group indices differ between 69 and 339 , whereas bandwidths change from $0.4 \mathrm{~nm}$ to $2.5 \mathrm{~nm}$ in this range. The normalized delay-bandwidth product is calculated as 0.085 for $\delta x=0.22 a$ and 0.114 for $\delta x=$ $0.27 a$. 


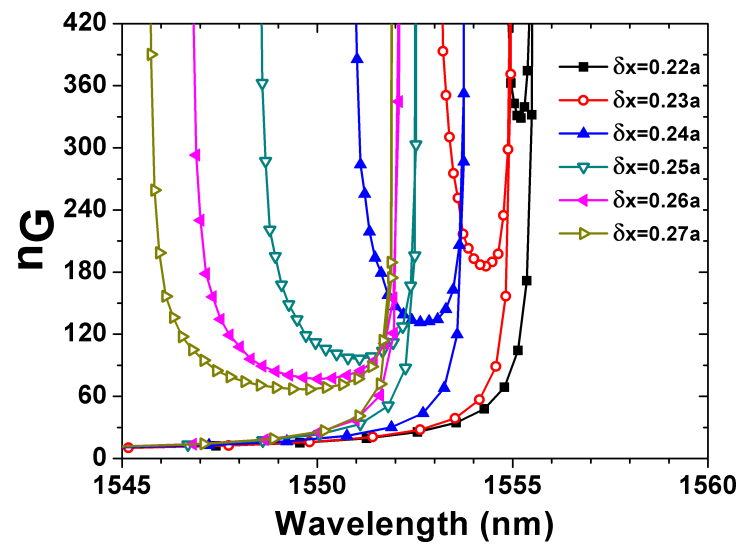

Fig. 4. Group index characteristics of the PC for $\delta x$ shifts between $0.22 a$ and $0.27 a$.

\subsection{Dynamic modulation performance of the photonic crystal slab}

The change of group index-wavelength curve as a function of modulated voltage is shown in Fig. 5 for the PC slab with $\delta x=0.22 a$. Substituting the average $n_{\mathrm{G}}$ value

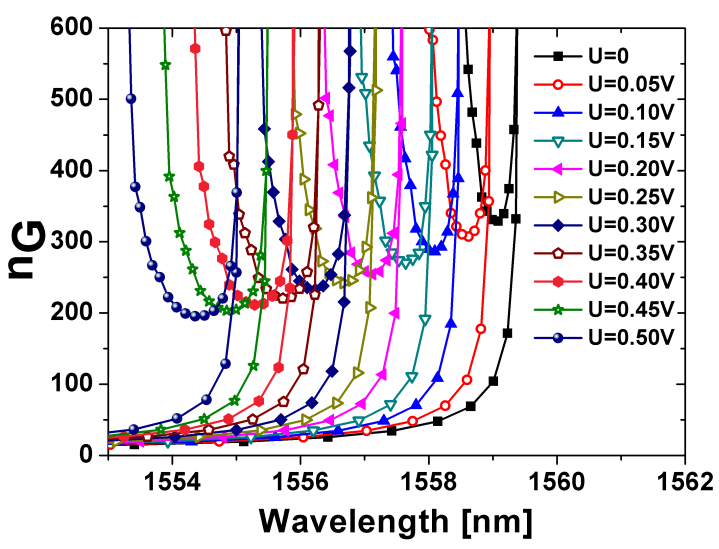

Fig. 5. Group index characteristics of the PC under voltage modulation.

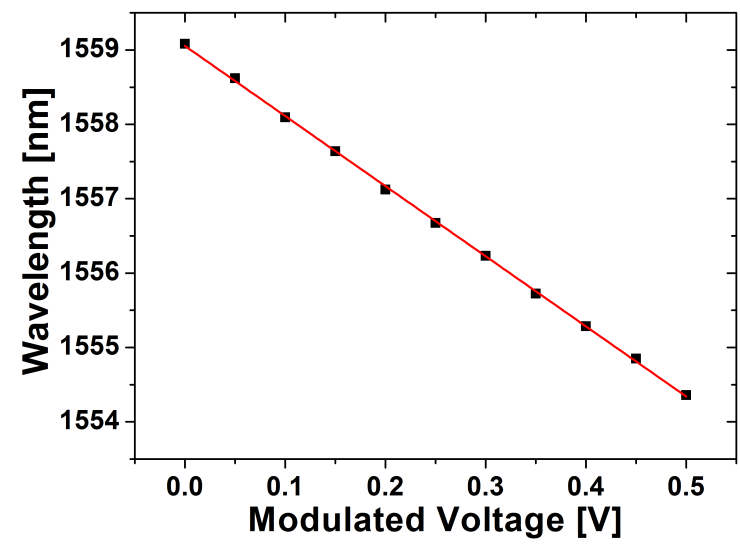

Fig. 6. Central wavelength of slow light region as a function of the modulated voltage. of 339.3 into Eq. (3) yields a local field factor as 14.56. As the applied voltage increases, the guided mode and the slow light region shifts to lower wavelengths. As the voltage increases to $0.5 \mathrm{~V}$, the average group index decreases to 201.5 and bandwidth increases to $0.8 \mathrm{~nm}$.

The change of central wavelength of slow light region as a function of voltage is shown in Fig. 6. The central wavelength shifts its spectral position following a linear behavior when a modulated voltage between $0 \mathrm{~V}$ and $0.5 \mathrm{~V}$ is applied. The modulation sensitivity is obtained as $9.45 \mathrm{~nm} / \mathrm{V}$. This value is more than one order of magnitude larger than that for the polymer infiltrated PC slab with adjusted rows of holes adjacent to the line-defect [13].

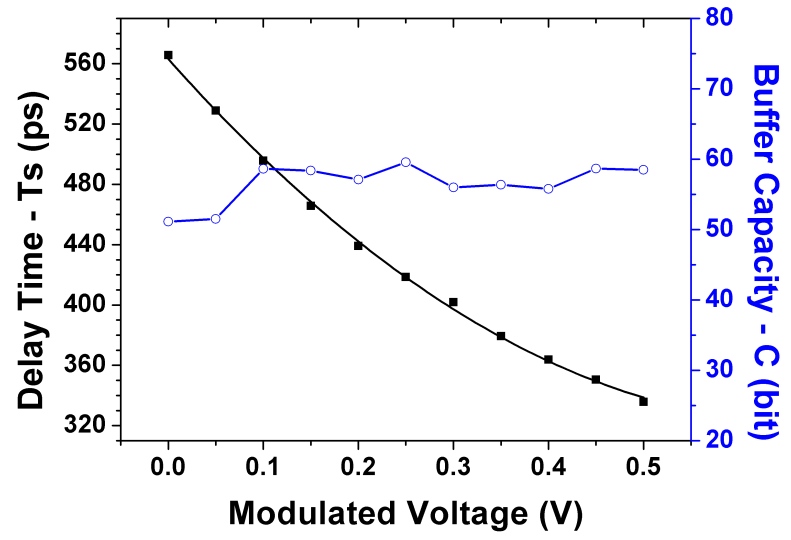

Fig. 7. Delay time and buffer capacity as a function of the modulated voltage $(L=0.5 \mathrm{~mm})$.

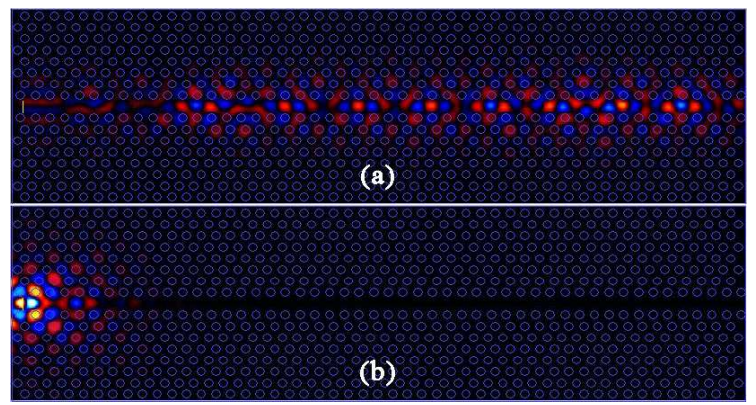

Fig. 8. Simulation of light propagation through the PC slab structure for $1.558 \mu \mathrm{m}$ wavelength at a fixed time for applied voltages of (a) $0.1 \mathrm{~V}$ and (b) $0.5 \mathrm{~V}$.

When the applied voltage changes from $0 \mathrm{~V}$ to $0.5 \mathrm{~V}$, the delay time decreases from $565.7 \mathrm{ps}$ to $335.8 \mathrm{ps}$, as shown in Fig. 7. However, under voltage change, the buffer capacity and the bit length are almost the same around 55 bit and $9 \mu \mathrm{m}$, respectively. Thus, the delay time can be controlled flexibly while keeping the buffer capacity and bit length almost fixed.

The wave propagation inside the $\mathrm{PC}$ slab is also simulated by finite-difference time-domain method [14] at different applied voltages to show the modulation property of the structure distinctly. The simulation results 
show that this PC slab design can be used as an on-off switch to store, release or adjust the timing of the optical signal. For example, as shown in Fig. 8, while light is delayed at $1.558 \mu \mathrm{m}$ when $0.1 \mathrm{~V}$ is applied, it can be stored in the PC based buffer when the modulated voltage is increased to $0.5 \mathrm{~V}$.

\section{Conclusions}

In this study, an electro-optic slow light modulator based on photonic crystal slab is proposed. The first rows adjacent to the waveguide are shifted by $0.22 a$ through the light propagation direction to slow down light by an order of 339 as compared to light velocity in vacuum. It is found that for this PC configuration, the guided mode shifts to lower wavelengths as voltage is applied to the structure. A linear change of slow light central wavelength with voltage is obtained and the modulation sensitivity is obtained as $9.45 \mathrm{~nm} / \mathrm{V}$. Flexible manipulation of storing, releasing, and tuning the delay of optical signal is demonstrated while keeping the buffer capacity and bit length almost fixed.

\section{Acknowledgments}

We gratefully acknowledge the financial support by Scientific Research Projects of Ankara University (BAP) under grant no. 12B4343011.

\section{References}

[1] R.S. Tucker, P.-C. Ku, C.J. Chang-Hasnain, J. Lightwave Technol. 23, 4046 (2005).

[2] T. Baba, Nature Photon. 2, 465 (2008).
[3] M. Soljacić, J.D. Joannopoulos, Nature Mater. 3, $211(2004)$

[4] N. Natomi, K. Yamada, A. Shinya, T. Takahashi, C. Takahashi, I. Yokohama, Phys. Rev. Lett. 87, 253902 (2001).

[5] L.H. Frandsen, A.V. Lavrinenko, J.F. Pefersen, P.I. Borel, Opt. Express 14, 9444 (2006).

[6] S. Kubo, D. Mori, T. Baba, Opt. Lett. 32, 2981 (2007).

[7] J.T. Li, T.P. White, L. O'Faolain, A. Gomez-Iglesias, T.F. Krauss, Opt. Expr. 16, 6227 (2008).

[8] R. Hao, E. Cassan, H. Kurt, J. Hou, X. Le Roux, D. Marris-Morini, L. Vivien, D. Gao, Z. Zhou, X. Zhang, IEEE Photon. Technol. Lett. 22, 844 (2010).

[9] J.D. Luo, Y.J. Cheng, T.D. Kim, S. Hau, S.H. Jang, Z.W. Shi, X.H. Zhou, A.K.-Y. Jen, Organic Lett. 8, 1387 (2006)

[10] S.G. Johnson, J.D. Joannopoulos, Opt. Expr. 8, 173 (2001).

[11] J.-M. Brosi, C. Koos, L.C. Andreani, M. Waldow, J. Leuthold, W. Freude, Opt. Expr. 16, 4177 (2008).

[12] L. Razzari, D. Trager, M. Astic, P. Delaye, R. Frey, G. Roosen, R. Andre, Appl. Phys. Lett. 86, 231106 (2005).

[13] F. Long, H. Tian, Y. Ji, J. Lightwave Technol. 28 , 1139 (2010).

[14] http://ww.photondesign.com/products/ crystalwave.htm. 